\title{
Gráfica de peso neonatal vs. edad gestacional en el Instituto Materno Infantil de Bogotá
}

\author{
Juan Carlos Sabogal*, Heidy Cáceres**
}

Recibido: Abril 11 / 2000

Revisado: Abril 17 / 2000

Aceptado: Agosto $11 / 2000$

\section{RESUMEN}

El retardo de crecimiento intrauterino es una de las causas más frecuentes de remisión a nuestro hospital. La clasificación adecuada del neonato es muy importante y es necesario un patrón comparativo pertinente. El objetivo del estudio es hallar una curva de peso vs edad gestacional a partir de sujetos normales. Se incluyó una población de 10.553 neonatos provenientes de madres sanas, con fecha de última al paquete estadístico STATA 6.0 y se determinaron los percentiles para cada edad gestacional entre 30 y 42 semanas. Luego se construyó una gráfica de peso vs edad gestacional que incluye la distribución de los percentiles 10, 25, 50, 75 y 90 . Se realiza una comparación entre la gráfica obtenida y la tradicionalmente utilizada de Lubchenko y se discuten las implicaciones que tendría el uso de la nueva gráfica en la clasificación de pequeño, adecuado o grande para edad gestacional.

PALABRAS CLAVES: Crecimiento fetal, retardo de crecimiento intrauterino, macrosomía fetal.

\section{SUMMARY}

Fetal growth retardation is among the commonest referal indications to our hospital. Propper classification is a must and an appropiate graphics to compare are needed. The aim of the study was to obtain a graphic of weight vs gestational age from normal subjects. 10.553 neonates were included from healthy mothers with reliable last mense. No pathologic condition was found (other than preterm labor). Data base were imported to the STATA 6.0 statiscal pack and percentils were determined for every gestational age from 30 to 42 weeks gestation.

Then a graphic of weight vs gestational age was obtained including the 10, 25, 50, 75 and 90th percentiles. Comparison with the clasic graphic from Lubchenko and implications for the classification as small, adecuate or large for gestational age are revised.

KEY WORDS: Fetal growth, intrauterine fetal growth retardation, macrosomia.

\section{Introducción}

La necesidad de contar con una gráfica de distribución percentilar de Peso vs Edad gestacional que sea representativa de la población estudiada es importante en un hospital que como el nuestro, se halla dedicado a la atención de embarazos de alto riesgo. Muchas de las pacientes remitidas a este centro, presentan complicaciones de potencial influencia sobre el peso fetal, tales como sospecha de retardo de crecimiento intrauterino, preeclampsia, diabetes gestacional, etc... (1) y por ende resulta necesario que el patrón de referencia para comparar el peso fetal de los recién nacidos sea racional. En nuestra institución, tradicionalmente se ha usado la gráfica de peso vs edad gestacional de Battaglia y Lubchenko (2), desarrollada a una altitud similar a la de Bogotá y comprendiendo un $30 \%$ de población fetal de origen hispano. En la práctica, aunque útil, supone percentiles algo extremos, teniendo en cuenta que el percentil 10 para 36 semanas es de 2.000 gramos. Sólo aquellos casos de retardo de crecimiento severo superan este umbral y son clasificados adecuadamente, "ignorando" fetos que sufren complicaciones perinatales propias del bajo peso pero que no alcanzan dicho límite.

Por otro lado, está claramente establecido en la literatura que el potencial de crecimiento fetal está sujeto a numerosas variables disímiles como la raza, la altura sobre el nivel del mar, factores genéticos, genéricos, ambientales, hábitos maternos, enfermedades maternas, administración de medicamentos, etc... (3), cuya influencia en nuestra población ha sido pobremente descrita. Es por ello que los patrones sólo deben aplicarse a sujetos de la población a partir de la cual se obtuvieron.

Finalmente, desde el punto de vista clínico resulta de capital importancia que la valoración ecográfica del crecimiento

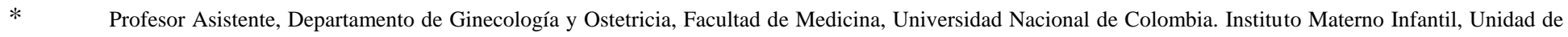
Ultrasonido, Bogotá.

Médica Interna. Internado Especial. Centro de Epidemiología Clínica, Facultad de Medicina, Universidad Nacional de Colombia, Instituto Materno Infantil, Bogotá.
} 
intrauterino, problema frecuente en nuestro hospital, no adolezca del sesgo que implica someter la biometría fetal a una distribución percentilar ajena a la población escrutada, sino que por el contrario provenga de una población equiparable. Tal es el propósito del presente trabajo.

\section{Materiales y métodos}

Desde 1995, se implementó en el Instituto Materno Infantil el Sistema Informático Perinatal CLAP-OPS, que comprende el registro sistemático de variables significativas propias del control prenatal, parto y puerperio inmediato, en un formato diseñado para tal fin (4). A partir de dicho formato se ha obtenido una base de datos considerable con la cual es posible cruzar diversas variables, entre ellas el peso al nacimiento vs edad gestacional. El peso es aquel obtenido por el pediatra inmediatamente después de ocurrido el nacimiento con el feto ya ligado y desnudo, en báscula mecánica con escala cada 20 gramos. La edad gestacional es aquella obtenida a partir de la fecha de última regla confiable (ciclos regulares, ausencia de planificación hormonal en los tres meses previos a la amenorrea y último período de características habituales y claramente identificable). Se obtuvieron datos de 31.926 partos desde
Febrero de 1995 hasta Diciembre de 1999, de los cuales 10.553 se catalogaron como "sanos", habida cuenta de la ausencia de enfermedad materna o fetal alguna asociada al embarazo o parto, que sirvieron como base para la distribución percentiar del presente trabajo. Tales partos incluyen indistintamente la vía vaginal o abdominal, feto vivo, sin patología perinatal identificable y evolución postnatal inmediata normal. Si bien una fracción de los recién nacidos pretérmino eventualmente pudieron tener hospitalización preventiva, no se identificó patología adicional a su condición de prematuros. La distribución percentilar se realizó a partir de las 30 semanas siempre que hubiera más de 30 pacientes para cada edad gestacional. Se calcularon los percentiles 10, 25, 50, 75 y 90 para cada edad gestacional. Los datos numéricos de los 10.675 partos fueron importados como base única de datos y analizados con el paquete estadístico STATA 6. Se realizó una distribución de frecuencias por semanas y posteriormente se obtuvo la gráfica de distribución percentilar.

Como puede observarse, se obtuvo una gráfica de distribución percentilar peso vs Edad gestacional, que al constituir un fenómeno esencialmente descriptivo, se presenta tal y como se obtuvo a continuación (ver tabla y gráfica 1).

\section{Resultados}

\begin{tabular}{|c|c|c|c|c|c|c|}
\hline \multirow[t]{2}{*}{ E.G. } & \multirow[t]{2}{*}{ \# DE CASOS } & \multicolumn{5}{|c|}{ PERCENTILES } \\
\hline & & 10 & 25 & 50 & 75 & 90 \\
\hline 30 & 42 & 1060 & 1375 & 1900 & 2775 & 3170 \\
\hline 31 & 50 & 1525 & 1731 & 2175 & 2858 & 3083 \\
\hline 32 & 60 & 1600 & 1800 & 2360 & 2800 & 3200 \\
\hline 33 & 76 & 1670 & 2000 & 2333 & 2650 & 2943 \\
\hline 34 & 145 & 1919 & 2194 & 2531 & 2867 & 3208 \\
\hline 35 & 253 & 2074 & 2346 & 2581 & 2884 & 3204 \\
\hline 36 & 580 & 2267 & 2521 & 2764 & 3046 & 3318 \\
\hline 37 & 1113 & 2457 & 2668 & 2913 & 3158 & 3409 \\
\hline 38 & 2196 & 2564 & 2797 & 3039 & 3291 & 3520 \\
\hline 39 & 2709 & 2634 & 2884 & 3132 & 3372 & 3618 \\
\hline 40 & 2300 & 2709 & 2956 & 3206 & 3454 & 3688 \\
\hline 41 & 788 & 2742 & 2983 & 3253 & 3481 & 3777 \\
\hline 42 & 241 & 2741 & 2966 & 3198 & 3480 & 3729 \\
\hline
\end{tabular}

Valores numéricos de la distribución percentilar de peso vs edad gestacional en el Instituto Materno Infantil de Bogotá 


\section{Gráfica $0016 \mathrm{i} 01$ \\ PESO VS. EDAD GESTACIONAL DISTRIBUCION PERCENTILAR OBTENIDA EN EL IMI, CON 10.553 NEONATOS. SE INCLUYEN LOS PERCENTILES 10, 25, 50, 75 y 90}

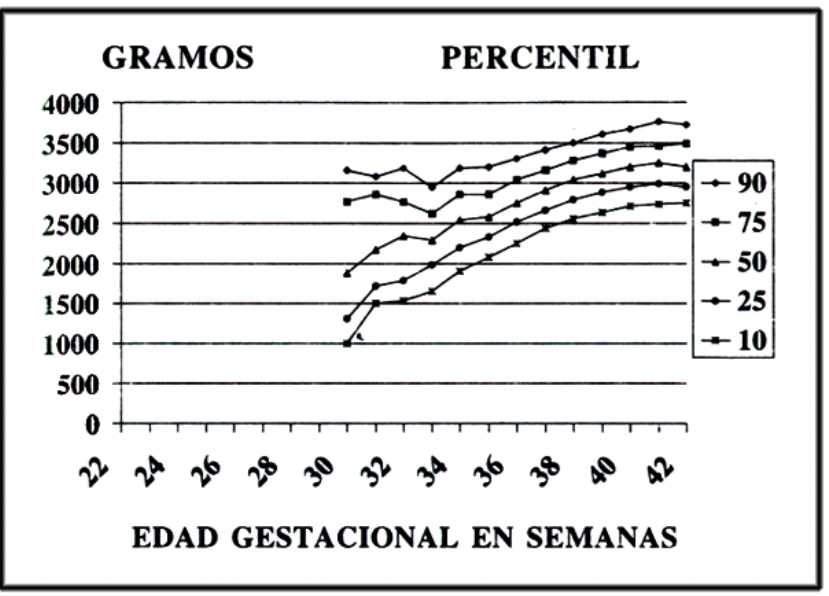

Análisis

Como era de esperarse, el peso fetal se incrementa con la edad gestacional. El análisis de los datos muestra que no tienen distribución normal. Los valores de los percentiles tanto superiores como inferiores tienden a ser mayores, con respecto a la gráfica de Lubchenko, particularmente entre las semanas 26 a 34. A partir de las 35 semanas, se observa una distribución percentilar similar aunque los percentiles inferiores tienden a ser algo mayores a los de la gráfica tradicional (gráfica 2). De lo anterior se desprende que el bajo peso al nacer puede haber sido subdiagnosticado al basarse en la gráfica tradicional (Lubchenko), principalmente al final del segundo e inicio del tercer trimestre. Al mismo tiempo se encuentra que el límite superior (percentil 90), para el diagnóstico de fetos macrosómicos es mayor a las mismas edades gestacionales. De modo similar, es posible que en lo sucesivo sea menor el diagnóstico de macrosomía fetal a edades gestacionales tempranas ( 26 a 34 semanas), dado que el peso necesario para el diagnóstico es mayor en nuestra tabla, si bien en los embarazos a término es prácticamente igual.

Adicionalmente, se aprecia un rango intercuartílico menor a edades gestacionales tardías con respecto a edades gestacionales tempranas. Lo que sugiere una menor dispersión por efecto de tamaño de la población ya que la mayoría de los datos corresponden a niños entre 37 y 40 semanas.
Gráfica $0016 \mathrm{i} 02$

COMPARACIÓN ENTRE LA DISTRIBUCION

PERCENTILAR OBTENIDA (___ $Y$ Y LA

CLASICAMENTE USADA DE LUBCHENKO (- . - - ). NOTESE UNA MARCADA DIFERENCIA A EDADES GESTACIONALES RELATIVAMENTE TEMPRANAS

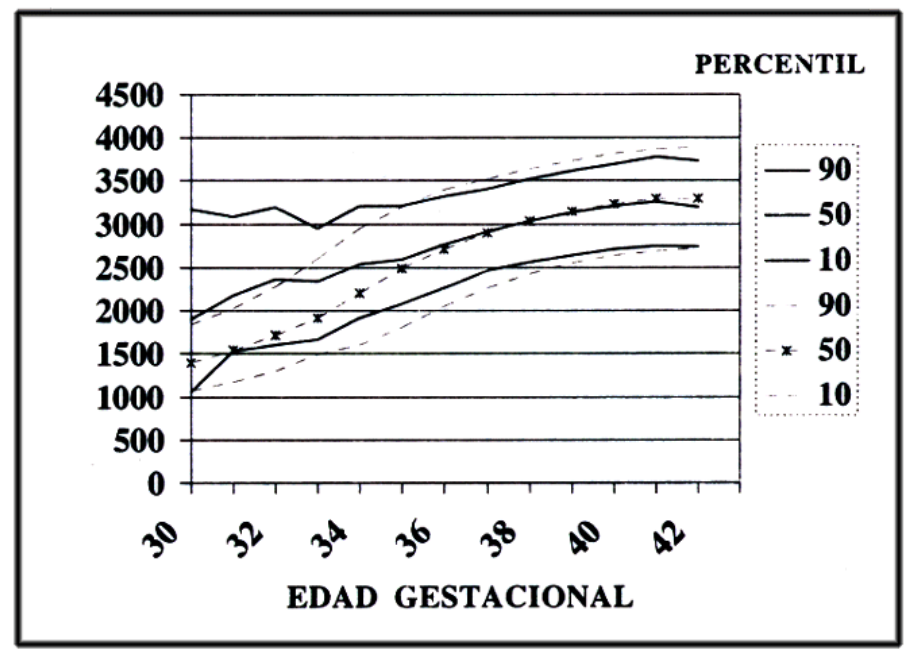

A pesar de que en la muestra hubo pacientes con edad gestacional temprana (menor de 30 semanas), su numero no permitió obtener una distribución percentilar con fiable, razón por la cual se omitieron de la gráfica presentada. Hacia el futuro, es importante señalar que procede el estudio poblacional con base en múltiples variables y no solamente comprendiendo la edad gestacional.

\section{Conclusiones}

El uso de una gráfica percentilar como la presentada es más adecuado al evaluar el peso para la edad gestacional dado que procede de datos de nuestra población.

El cambio que se observa es más evidente a las edades gestacionales entre las 26 y las 34 semanas, en que los pesos exhibidos por la nueva gráfica son mayores en todos los percentiles al compararlos con la tradicional (Lubchenko). La diferencia en los embarazos a término entre las dos gráficas es mínima.

Las entidades patológicas involucradas en el peso fetal y al nacer como el retardo de crecimiento intrauterino o la macrosomía fetal serán más adecuadamente diagnosticados con esta nueva gráfica.

Se desea reconocer y agradecer la invaluable ayuda del Centro de Epidemiología Clínica de la Facultad de Medicina de la Universidad Nacional de Colombia cuya asesoría hizo posible el presente trabajo.

\section{BIBLIOGRAFIA}

1. Comité de Mortalidad materna. Reporte anual del Comité de Mortalidad del Instituto Materno Infantil, Bogotá, 1998.

2. Battaglia FC, Lubchenko MO. A practical classification of newborn infants by weight and gestational age. J Pediatrics 1967; 71(2): 159-163.
3. Creasy RK, Resnik R. Maternal-Fetal Medicine. Principles and practice. Third Edition. W. B. Saunders Company, 1994.

4. Polin RA, Fox WW. Fetal and Neonatal Physiology. W. B. Saunders Company, 1992.

5. Fescina R, Schwarcz R. Manual de técnicas perinatales. Centro Latinoamericano de Perinatología, Montevideo, 1992. 